\title{
BANKWIRTSCHAFTLICHE SCHRIFTENREIHE
}

Herausgegeben von Professor Dr. H. Linhardt 
Horst Pastuszek

\section{WIRTSCHAFTSORDNUNG \\ UND WIRTSCHAFTSPUBLIZISTIK}

INSBESONDERE BANKENPUBLIZISTIK

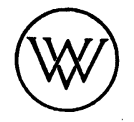

SPRINGER FACHMEDIEN WIESBADEN GMBH 
ISBN 978-3-663-04024-8 ISBN 978-3-663-05470-2 (eBook) DOI $10.1007 / 978-3-663-05470-2$

(C) 1959 Springer Fachmedien Wresbaden

Ursprünglich erschienen bei Westdeutscher Verlag, Koln und Opladen 1959 


\section{VORWORT}

Jede wissenschaftliche Arbeit, die sich mit den Grundordnungen des wirtschaftlichen Lebens befaßt, stößt früher oder später an jene Grenze, welche die objektiv-wissenschaftliche Aussage von der subjektiv-wertenden professio trennt, so auch die vorliegende Untersuchung. Wenn diese Grenze an einigen Stellen bewußt überschritten wurde, so glaube ich, daß dadurch eine saubere wissenschaftliche Arbeit keine Einbuße erfährt, sondern daß im Gegenteil ein verantwortungsbewußtes und wohlfundiertes Bekenntnis von einem Wissenschaftler erwartet werden kann, solange nur die Grenzen zwischen beiden Bereichen nicht verwischt werden. Darum aber habe ich mich eifrig bemüht, indem ich die Worte so gewählt habe, daß beim Leser kein Zweifel bleiben kann, welchem Bereich die jeweiligen Ausführungen zuzuordnen sind.

Ich war bestrebt, gewissenhaft sowohl diejenigen Quellen zu nennen, auf denen bestimmte Aussagen basieren, und sei es nur, daß sie den zündenden Gedanken abgegeben haben, als auch diejenigen, die die eigenen Gedanken bekräftigen. Eines aber war nicht möglich, die geistige Abhängigkeit in der Untersuchung selbst zum Ausdruck zu bringen, die ich gegenüber mehreren wissenschaftlichen Lehrern und Autoren empfinde, ohne die diese Arbeit nicht in gleicher Weise entstanden wäre, vor allem die geistige Abhängigkeit, die ich freimütig und dankbar gegenüber meinem hochverehrten akademischen Lehrer, Herrn Professor Dr. Hanns Linbardt, seinerzeit Ordinarius für Betriebswirtschaftslehre und Direktor des Seminars für Bank- und Kreditwirtschaft der Freien Universität Berlin, jetzt an der Hochschule für Wirtschafts- und Sozialwissenschaften Nürnberg, bekenne; denn »im Innersten wirkende geistige Abhängigkeit ist ein für allemal kein Thema für Anmerkungen, da sie sich nicht mit einem Citat oder einem Stellennachweis mit Band- und Seitenzahl abmachen läßt « (Johann Plenge, in den Vorbemerkungen zu seinem "System der Verkehrswirtschaft «). Was ich in einem halben Jahrzehnt von dem Wissen, der Lebenserfahrung und dem beispielhaften, un- 
erschütterlichen Mut zur Wahrheit in den Vorlesungen im Auditorium, in den Diskussionen im Seminar, in den vielen persönlichen Gesprächen und der engen Mitarbeit als Student und vor allem als wissenschaftlicher Assistent von Herrn Professor Dr. Linhardt bewußt oder unbewußt übernehmen durfte, verpflichtet mich zu tiefem Dank. Das Geben des Lehrers und das Nehmen des Schülers entbindet mich nicht davon, für das geschriebene Wort die alleinige Verantwortung zu tragen.

Die Arbeit wurde als Dissertation im Januar 1956 der Wirtschaftsund Sozialwissenschaftlichen Fakultät der Freien Universität Berlin eingereicht. Als Erstgutachter wurde von der Fakultät Herr Prof. Dr. Hanns Linhardt und als Zweitgutachter Herr Prof. Dr. Friedrich Bülow bestellt. Es ist mir ein Bedürfnis, auch an dieser Stelle Herrn Prof. Dr. Bülow für seine wohlwollende Förderung ergebenst zu danken.

Die vorliegende Fassung der Untersuchung unterscheidet sich von der Dissertation nur unwesentlich. Sie wurde lediglich anders gegliedert und durch inzwischen erforderlich gewordene Nachträge ergänzt.

Im ersten Hauptteil wird der Versuch unternommen, theoretischsystematisch nach einer allgemeinen Grundlegung die Beziehungen zwischen den Wirtschaftsordnungen und der Wirtschaftspublizistik zu ergründen, die Funktionen, die Bedeutung und die Gestaltung der Wirtschaftspublizistik in den einzelnen Wirtschaftsordnungen aufzuzeigen und abschließend eine Systematik der Wirtschaftspublizistik zu gewinnen. Die von den Wirtschaftssubjekten selbst gepflegte Wirtschaftspublizistik wird mit einer gewissen Betonung behandelt, ohne $\mathrm{da} ß$ eine eindeutige, dahingehende Schwerpunktverlagerung vorgenommen wird.

Der Untertitel der vorliegenden Arbeit ist für diesen Teil der Untersuchung ohne besonderes Gewicht, unbeschadet dessen, daß die Wirtschaftspublizistik als Ganzes auch die Bankenpublizistik als Teil einschließt.

Ein solcher Versuch liegt der Natur der Sache nach vorwiegend im rein Theoretischen begründet und bietet daher den Reiz, den das Durchdringen komplexer Probleme im Wege abstrakter Denkarbeit demjenigen eröffnet, der sich ihr um der Erkenntnis willen unterzieht.

Der zweite Hauptteil dient der Verifikation und Ergänzung des theoretischen Teils der Arbeit, ausgehend von den Realtypen der Wirtschaftsordnungen. Es wäre durchaus möglich, die Verifikation 
auf das ganze System der Wirtschaftspublizistik zu erstrecken. Bei einer solchen Zielsetzung müßten jedoch zwei Nachteile in Kauf genommen werden: Einerseits würden auch diejenigen Teile des Systems einer Darstellung der Fakten zu unterziehen sein, die bereits hinreichend in der Literatur behandelt oder aus der täglichen Erfahrung her bekannt sind, und andererseits würden die einzelnen Teile des Systems notwendig in einer Kürze abzuhandeln sein, die nur in einer theoretischen Studie mit Erfolg zu erzielen ist. Zu dieser Feststellung tritt eine weitere wesentliche Erkenntnis hinzu: Besonders eine Gliedkette im System der Wirtschaftspublizistik hat bisher in der Literatur keine geschlossene und systematisch eingepaßte Würdigung gefunden - die informierende Publizistik als Nebenleistung der Kreditinstitute, obwohl sie in einer Entwicklung von sechs Jahrzehnten zu einem differenzierten und spezialisierten integrierenden Bestandteil der Wirtschaftspublizistik gewachsen ist.

Diese Überlegungen gaben den Anlaß, die Publizistik der Banken zum Hauptgegenstand des zweiten Teiles der Arbeit zu machen, zumal sie besondere Aspekte im Vergleich zwischen der Verkehrswirtschaft und der Zentralverwaltungswirtschaft eröffnet. Gleichzeitig findet die Aufnahme des Buches in die »Bankwirtschaftliche Schriftenreihe« darin ihre Rechtfertigung. Die Wahl des Untertitels wird durch den zweiten Teil der Arbeit hinreichend begründet, wie auch dieser durch jenen angemessen zum Ausdruck kommt.

Mit der Darstellung der informierenden Bankenpublizistik wird insofern Neuland betreten, als bisher nur in wenigen Fällen in der wissenschaftlichen Literatur von der Bankenpublizistik Notiz genommen wurde und dann vorwiegend nur in $\operatorname{der}$ Weise, daß die Bankenpublikationen als Quellen gedient haben.

Der Weg war somit vorgezeichnet, der beschritten werden mußte und der prinzipiell immer der gleiche ist, wenn Neuland betreten wird: Es ist die Sammlung und Ordnung des Einzelmaterials und die darauf aufbauende, soweit als möglich bis ins Detail gehende Deskription - in erster Linie die Vermittlung der Kenntnis des Tatsächlichen und erst in zweiter Linie der Erkenntnis des Wesentlichen. Ein erster, unumgänglicher Schritt, in gewisser Weise eine Kärrnerarbeit, die wie diese undankbar ist, die aber einmal geleistet werden muß. Derjenige, der wissenschaftlich zu arbeiten gewohnt ist, tut lieber den zweiten Schritt - auch ich hätte ihn gern getan, wenn es nicht der Voraussetzungen gemangelt hätte, die ich nun zu schaffen bestrebt war. Nichtsdestoweniger gebe ich der Hoffnung Ausdruck, 
daß auch dieser Teil der Arbeit dem Leser, insbesondere dem an der Bankwirtschaft interessierten und dem in der Bankpraxis stehenden Leser, etwas zu sagen haben wird.

Vielen in- und ausländischen Banken sei an dieser Stelle für freundlichst erteilte Informationen gedankt.

Meiner Frau Edith schulde ich Dank für ihre verständnisvolle Hilfe, die sie mir in oft nächtelanger Arbeit durch das Schreiben des Manuskriptes vom ersten Entwurf bis zu den mehrmaligen Überarbeitungen gewährt hat.

Offenburg/Baden, im Herbst 1958

Horst Pastuszek 


\section{INHALTSVERZEICHNIS}

\section{Einleitung}

1. Zur Tatsachenfindung (Erfahrungsobjekt). . . . . . . . . . . 1

2. Bestimmung des Erkenntnisobjektes . . . . . . . . . . . 3

1) Abgrenzungen . . . . . . . . . . . . . . . 3

2) Erkenntnisobjekt . . . . . . . . . . . . . 4

\section{ALLGEMEINE GRUNDLEGUNG}

A. Etymologische und analytische Bestimmung der Begriffe

1. Publizistik, Publizist . . . . . . . . . . . . . . . . . . . . . 9

1) Sprachgeschichtliche Wandlungen der Begriffe . . . . . . . . 9

2) Wissenschaftliche Definitionen und Begriffsmerkmale . . . . . 11

aa) Aussage . . . . . . . . . . . . . . . . . . . . . . . . . . 13

bb) öffentlich . . . . . . . . . . . . . . . . . . . . . . . 13

cc) Bewußtseinsinhalte . . . . . . . . . . . . . . . . . . . 14

dd) aktuell . . . . . . . . . . . . . . . . . . . . . . . . . . . 14

2. Publikum . . . . . . . . . . . . . . . . . . . . . . . . . 14

1) Das Wort im Sprachgebrauch . . . . . . . . . . . . . . 14

2) Der Begriff in der wissenschaftlichen Literatur . . . . . . . . 15

3) Analytische Bestimmung des Begriffs . . . . . . . . . . . . 17

3. Öffentlichkeit . . . . . . . . . . . . . . . . . . . 20

4. Publizität. . . . . . . . . . . . . . . . . . . . . . 22

1) Zustand . . . . . . . . . . . . . . . . . . . . . . . . 22

2) Tätigkeit . . . . . . . . . . . . . . . . . . . . . . . . 22

3) Eigenschaft - Originäre und derivative Publizität . . . . . . . 22

4) Effektive und latente Publizität . . . . . . . . . . . . . . . 23

5. Publikation . . . . . . . . . . . . . . . . . . 23

6. publizieren . . . . . . . . . . . . . . . . . . . . 24

7. öffentlich . . . . . . . . . . . . . . . . . . 25 
B. Die publizistischen Beziehungsfelder

1. Einführung . . . . . . . . . . . . . . . . . . . . . . 27

2. Graphische Darstellung der Beziehungsfelder . . . . . . . . . . . 27

a) Originäre Publizität . . . . . . . . . . . . . . . . . . . . . . . . . . . . 29

1) Unmittelbare Beziehung - direktes Interesse . . . . . . . . . 29

2) Unmittelbare Beziehung - indirektes Interesse . . . . . . . . 29

3) Unmittelbare Beziehung - direktes Interesse . . . . . . . . . 30

4) Unmittelbare Beziehung - indirektes Interesse . . . . . . . . 30

b) Derivative Publizität . . . . . . . . . . . . . . . . . . . . 31

5) Unmittelbare Beziehung - direktes Interesse . . . . . . . . . 31

6) Unmittelbare Beziehung - indirektes Interesse . . . . . . . . 31

7) Mittelbare Beziehung-direktes Interesse . . . . . . . . . 32

8) Mittelbare Beziehung-indirektes Interesse . . . . . . . . 32

\section{THEORETISCH-SYSTEMATISCHER HAUPTTEIL}

\section{Einführung}

1. Zur Wirtschaftspublizistik. . . . . . . . . . . . . . . . . . . 37

1) Potentiell öffentliche und private Bereiche . . . . . . . . . . 37

2) Wirtschaft und Publizistik . . . . . . . . . . . . . . . . 38

aa) Die Wirtschaft als Grenzgebiet der Publizistik . . . . . . . 38

bb) Privatökonomie und Publizistik . . . . . . . . . . . . . . . . 38

cc) Sozialökonomie und Publizistik . . . . . . . . . . . . . . . . . 38

3) Qualifizierte und unqualifizierte Publizistik . . . . . . . . . 38

2. Zur Wirtschaftsordnung . . . . . . . . . . . . . . . . . . 39

A. Zentralgeleitete Wirtschaft und Wirtschaftspublizistik

I. Eigenwirtschaft . . . . . . . . . . . . . . . . . . . . . . . . 41

II. Totale Zentralverwaltungswirtschaft . . . . . . . . . . . . . . . 42

1. Zwei ungelöste Probleme der zentralen Planung . . . . . . . . . 44

2. Rationalität und Wirtschaftsrechnung . . . . . . . . . . . . . . 48

1) Formale und materiale Rationalität . . . . . . . . . . . . 48

2) Drei Rechnungssysteme . . . . . . . . . . . . . . . . . . . 49

3) Naturalrechnung . . . . . . . . . . . . . . . . . . . . . . . . . . . 49

4) Rechnung mit mathematisch

5) Resümee . . . . . . . . . . . . . . . . . . . . . . . 52

3. Die Folgen der Unlösbarkeit der Probleme. . . . . . . . . . . . 53 
1) Relativ niedriges Versorgungsniveau . . . . . . . . . . . 53

2) Engpässe, Friktionen und konjunkturelle Schwankungen . . . 54

3) Widerstände der Konsumenten . . . . . . . . . . . . . . . 55

4) Wirtschaftlicher und politischer Zwang . . . . . . . . . . 56

4. Die Publizistik als politisches Führungsmittel. . . . . . . . . . 57

1) Die Funktionen der Publizistik . . . . . . . . . . . . . 57

2) Unqualifizierte Publizistik als Teil der Propaganda . . . . . . 58

III. Zentralverwaltungswirtschaft mit freiem Konsumguttausch . . . . . 58

1. Die Stellung der Publizistik im Vergleich zur totalen

Zentralverwaltungswirtschaft . . . . . . . . . . . . . . . 58

2. Die originäre Publizität der Tauschmärkte und die unentwickelte Publizistik der Konsumenten . . . . . . . . . . . . . . . . . 59

IV. Zentralverwaltungswirtschaft mit freier Konsumwahl. . . . . . . . 60

1. Möglichkeiten der Wirtschaftsrechnung . . . . . . . . . . . . 60

1) Die beiden bereits abgehandelten Rechnungssysteme . . . . . 60

2) Die Rechnung mit Näherungswerten auf Grund der Nachfrageund Angebotssituation am Konsumgütermarkt . . . . . . . 61

aa) Die Antagonie bei freier Preisbildung . . . . . . . . . 61

bb) Die Problematik bei autonomer Preisfixierung . . . . . 62

2. Die Reaktionsmöglichkeiten der Planbehörde auf Konsumentenpläne 63

1) Aktive Reaktion. . . . . . . . . . . . . . . . . . . . . . 64

2) Passive Reaktion . . . . . . . . . . . . . . . . . . 64

3. Die Folgen der Situation und die Funktionen der Publizistik . . . 64

4. Originäre Publizität auf den Konsumgütermärkten und »Konsumlenkung « . . . . . . . . . . . . . . . . . . . . . . . . . . . . . 65

5. Zusammenfassung. . . . . . . . . . . . . . . . . . 66

B. Verkehrswirtschaft und Wirtschaftspublizistik

Einführung

1. Wirtschaftsrechnung . . . . . . . . . . . . . . . 68

2. Bildung der Marktformen. . . . . . . . . . . . . . . . . 71

3. Bedingungen des vollkommenen Marktes . . . . . . . . . . . 74

1) Homogenitätsprinzip . . . . . . . . . . . . . . . . . . . 74

2) Weitere Bedingungen. . . . . . . . . . . . . . . . . . 75

3) Markttransparenz . . . . . . . . . . . . . . . . . . . . 76

4. Informierende und werbende Publizistik . . . . . . . . . . . . 77

1) Eigenbezogene und fremdbezogene informierende Publizistik . 77

2) Reklame und Public-Relations-Publizistik . . . . . . . . . 77

3) Hauptzwecke der informierenden und werbenden Publizistik . 77 
I. Vollständige Konkurrenz ． . . . . . . . . . . . . . . . . 79

1. Reklame . . . . . . . . . . . . . 80

2. Public-Relations-Publizistik . . . . . . . . . . . 82

3. Informierende Publizistik . . . . . . . . . . . . . . . 82

4. Markttransparenz und Publizität . . . . . . . . . . . 82

5. Exkurs . . . . . . . . . . . . . . . . . 83

II. Polypolistische Konkurrenz . . . . . . . . . . . . . . . . . . . 85

1. Kräfte und Gegenkräfte der Unvollkommenheit des Marktes . . . 86

2. Funktionen und Grenzen der Reklame . . . . . . . . . . . . . 87

1) Publizitätsfunktion . . . . . . . . . . . . . . . . . . 87

2) Differenzierungsfunktion. . . . . . . . . . . . . . . . 87

3) Volkswirtschaftliche Beurteilung . . . . . . . . . . . . 89

3. Public-Relations-Publizistik . . . . . . . . . . . . . . . . . . 91

1) Wettbewerbswirtschaftliche Zwielichtigkeit und privatwirtschaftlicher Sinn der Public Relations . . . . . . . . . 91

2) Die speziellen Mittel der Public-Relations-Publizistik . . . . 92

3) Die Publika . . . . . . . . . . . . . . . . . . . . . . 94

4. Informierende Publizistik . . . . . . . . . . . . . 95

a) Endogene informierende Publizistik . . . . . . . . . . 95

1) Informierende Publizistik als Marktleistung gegen Entgelt . . 95

2) Informierende Publizistik als unentgeltliche Nebenleistung . . 96

3) Informierende Publizistik als Pflichtleistung (Publizitätspflicht) 98

b) Exogene informierende Publizistik . . . . . . . . . . . . . 100

1) Informierende Publizistik der Körperschaften der wirtschaftlichen Selbstverwaltung, Verbände und Interessenvertretungen . 100

2) Informierende Publizistik der öffentlich-rechtlichen Rundfunk- und Fernsehanstalten . . . . . . . . . . . . . 101

3) Informierende Publizistik der staatlichen Behörden, Ämter und ihnen gleichgestellten Institutionen . . . . . . . . . 101

4) Informierende Publizistik der Enquête-Kommissionen . . . . 103

5) Informierende Publizistik der inter- und supranationalen Organisationen und Institutionen . . . . . . . . . . . 104

6) Informierende Publizistik der Forschungsinstitute . . . . . . 104

5. Markttransparenz - Gesamtheitswissen und Einzelsonderwissen . . 105

a) Gesamtheitswissen - Originäre und derivative Publizität . . . . 105

b) Einzelsonderwissen und seine Quellen . . . . . . . . . . 105

1) Eigene Erfahrung und Beobachtung - unmittelbares Kontaktwissen . . . . . . . . . . . . . . . . 105

2) Berichterstattung der "Betriebspersonen« mittelbares Kontaktwissen . . . . . . . . . . . . . . . . 105 
3) Nichtöffentliche Mitteilungen insbesondere kaufmännische Korrespondenz . . . . . . . . 106

4) Marktforschung (Marktanalyse und Marktbeobachtung) Wissenschaftliche Markterkundung . . . . . . . . . 108

5) Gewerblich erteilte Auskünfte (Auskunfteien) und geschäftsfreundliche Auskünfte (insbesondere Bankauskünfte) . 109

6) Eigene Forschungs- und Entwicklungsarbeiten . . . . . . . 111

c) Beziehungen zwischen Einzelsonderwissen und Gesamtheitswissen sowie die Umsetzungsfunktion der Publizistik . . . . . . . . . 112 III. Angebotsoligopoloid und Nachfrageoligopoloid (Unvollkommene Oligopole). . . . . . . . . . . . . . . . . 113

1. Oligopolistische Verhaltensweise im Hinblick auf die Publizistik . . 114

1) Oligopolistische Interpendenz und Publizistik . . . . . . . . 114

2) Erwartungsstrukturen . . . . . . . . . . . . . 115

3) Reaktionsfreie Zone für alle marktpolitischen Maßnahmen . . 116

2. Die Bedeutung der Publizistik, insbesondere der Reklame, als marktpolitische Maßnahme . . . . . . . . . . . . 116

1) Intensität und Hypertrophie der Reklame (unter Berücksichtigung der Markenartikelreklame) . . . . . . . 117

2) Die Publizistik als Mittel des Wirtschaftskampfes . . . . . . 117 IV. Angebotsmonopoloid und Nachfragemonopoloid (unvollkommene Monopole) . . . . . . . . . . . . . . . . . . 118

1. Zur Reklame des Monopolisten . . . . . . . . . . . . . . . . 119

1) Die speziellen Funktionen und die Bedeutung der Reklame . . 119

2) Die Richtungen und die Grenzen der Reklame . . . . . . . 119

2. Monopole und Public Relations . . . . . . . . . . . . 120

C. Systematik der verkehrswirtschaftlichen Publizistik

1. Allgemeine Vorbemerkungen . . . . . . . . . . . . . . . . 122

2. Zu den Untersuchungs- und Darstellungsmethoden . . . . . . . . 124

1) Untersuchungsmethoden . . . . . . . . . . . . . . . 125

2) Darstellungsmethoden . . . . . . . . . . . . . . 125

3. Systematische Darstellung der Wirtschaftspublizistik in der Verkehrswirtschaft . . . . . . . . . . . . . 126

D. Statistische Ergänzungen

1. Tageszeitungen . . . . . . . . . . . . . 130

2. Radiogeräte. . . . . . . . . . . . . . . 132

3. Fernsehgeräte . . . . . . . . . . . . . . 133 
4. Filmtheater - Filmbesucher . . . . . . . . . . . . . . . 134

5. Werkzeitschriften . . . . . . . . . . . . 134

6. Briefpost . . . . . . . . . . . . . . 136

7. Telegramme . . . . . . . . . . . . . . . . 137

8. Fernsprechanschlüsse. . . . . . . . . . . . . 138

\section{EMPIRISCHER HAUPTTEIL}

\section{A. Ergänzungen und Exempel aus dem Realtyp der Zentralverwaltungswirtschaft}

1. Nochmals» sozialistische Wirtschaftsrechnung «. . . . . . . . . . 143

1) Schein und Sein . . . . . . . . . . . . . . . . . . 143

2) Die Kontrollfunktion der Wirtschaftsrechnung in der UdSSR . 144

2. Kollektiveigentum und Publizität - Ein Paradoxon . . . . . . 146

3. Zur»Reklame« in der UdSSR. . . . . . . . . . . . . . . . . 147

4. Entwicklung, Struktur, Wesens- und Funktionswandel der Banken, insbesondere in der UdSSR . . . . . . . . . . . . . 148

1) Verstaatlichung der Banken in der sozialistischen Wirtschaft. . 148

2) Entwicklung des Banksystems in der UdSSR . . . . . . . . 150

3) Gegenwärtige Struktur des sowjetischen Banksystems . . . . 152

4) Wesens- und Funktionswandel der Banken . . . . . . . . . 152

5) Bankenpublizistik? . . . . . . . . . . . . 155

B. Die Publizistik der Banken in der Verkehrswirtschaft

1. Die oligopoloide Marktform der Kreditmärkte, dargestellt am deutschen Kreditsystem, und ihr Einfluß auf die Bankenpublizistik . 159

1) Die Situation auf den nationalen, regionalen und lokalen Kreditmärkten . . . . . . . . . . . . . . . . . . 159

aa) Nationaler Kreditmarkt . . . . . . . . . . . . . . . . 159

bb) Regionale Kreditmärkte . . . . . . . . . . . . . . . . 159

cc) Lokale Kreditmärkte . . . . . . . . . . . . . . . . . 160

2) Die einseitige Geschlossenheit der Angebotsund Nachfrageoligopoloide . . . . . . . . . . . . . . . . 161

3) Das preispolitische Kollektivmonopol (Zins- und Konditionenkartell) . . . . . . . . . . . . . . 162

4) Die Verlagerung des Wettbewerbs auf andere marktpolitische Mittel unter Einfluß des Wettbewerbsabkommens . . . . . . 164

2. Die fremdbezogene informierende Publizistik der Banken . . . . 165 
a) Einführung und Abgrenzung. . . . . . . . . . . . . . . 165

b) Fremdbezogene informierende Publizistik der Geschäftsbanken . 167

1) Die historischen Anfänge . . . . . . . . . . . . . 168

aa) Bulletin der Schweizerischen Kreditanstalt . . . . . . 168

bb) Bulletin des Schweizerischen Bankvereins . . . . . . . . 171

cc) Monthly Letter der First National City Bank of New York 174

dd) Zur allgemeinen historischen Entwicklung . . . . . . 175

2) Berichte . . . . . . . . . . . . . . . . 176

aa) Zeitschriftenähnliche Wirtschaftsberichte . . . . . 177

bb) Zeitungsähnliche Wirtschaftsberichte . . . . . . . . . . 184

cc) Auslandsberichte . . . . . . . . . . . . . . . . . . . 190

dd) Länderberichte . . . . . . . . . . . . . . . 193

ee) Börsenberichte . . . . . . . . . . . . . . . . . 194

3) Mitteilungen, insbesondere Außenhandels-Mitteilungen . . . . 198

4) Zur Auflagenhöhe der Geschäftsbankpublikationen . . . . . 200

c) Fremdbezogene informierende Publizistik der Zentralnotenbanken 203

1) Gemischte Bulletins . . . . . . . . . . . . . . . 203

2) Statistische Bulletins . . . . . . . . . . . . . . . 210

3) Mitteilungen . . . . . . . . . . . . . . . . . . 210

4) Jahres-(Geschäfts-)berichte und Jahrbücher . . . . . . . . 211

d) Fremdbezogene informierende Publizistik der internationalen

Kreditinstitutionen . . . . . . . . . . . . . . 212

1) Bank für Internationalen Zahlungsausgleich . . . . . . . . 212

2) International Bank for Reconstruction and Development

(World Bank) . . . . . . . . . . . . . . . . . 212

3) International Monetary Fund . . . . . . . . . . . . . . . 212

Literaturverzeichnis . . . . . . . . . . . . . . . . 215

Personenverzeichnis . . . . . . . . . . . . . . . . 225

Institutionen- und Firmenverzeichnis . . . . . . . . . . . . . . . 228

Sachverzeichnis . . . . . . . . . . . . . . 232 